Koken, Mete; Gogus, Mustafa

Time Evolution of the Horseshoe Vortex System Forming Around a Bridge Abutment

Verfügbar unter / Available at:

https://hdl.handle.net/20.500.11970/100281

Vorgeschlagene Zitierweise / Suggested citation:

Koken, Mete; Gogus, Mustafa (2010): Time Evolution of the Horseshoe Vortex System Forming Around a Bridge Abutment. In: Burns, Susan E.; Bhatia, Shobha K.; Avila, Catherine M. C.; Hunt, Beatrice E. (Hg.): Proceedings 5th International Conference on Scour and Erosion (ICSE-5), November 7-10, 2010, San Francisco, USA. Reston, Va.: American Society of Civil Engineers. S. 668-677. 


\title{
Time Evolution of the Horseshoe Vortex System Forming Around a Bridge Abutment
}

\author{
Mete Koken, and Mustafa Gogus
}

Civil Engineering Department

Middle East Technical University; Ankara

Turkey; email: mkoken@metu.edu.tr

\begin{abstract}
Scour forming around an isolated bridge abutment with curved end is investigated in this study. Clear water scour experiments are conducted for an abutment in a $25 \mathrm{~m}$ long sediment flume at a Reynolds number of 45000. 3D scour patterns forming around the abutments are obtained using an array of acoustic transducers along a grid refined up to $1-2 \mathrm{~cm}$ spacing at different stages of the scour. Detached Eddy Simulation (DES) is performed at the same channel Reynolds number for flat bed case (initiation of the scour) and for two deformed bed cases (intermediate stages of scour). Incoming flow in the simulations were fully turbulent containing unsteady velocity fluctuations. Variations in the structure and intensity of the horseshoe vortex system are investigated. At the initial stage of the scour the main and secondary horseshoe vortices undergo aperiodic bimodal oscillations. Those oscillations cause the horseshoe vortices to induce large bed shear stress values beneath them. As the scour hole starts forming secondary necklace vortex, HV2, gets closer to the primary necklace vortex, HV1, and merges with it at a location close to the abutment tip.
\end{abstract}

\section{INTRODUCTION}

Bridge abutments cause a very complex 3D flow field to occur within its proximity. The incoming boundary layer separates as the abutment is approached in the presence of the adverse pressure gradients. As a result of this separation, necklace shaped vortical structures (so called horseshoe vortex system) form around base of the abutment. When the abutment lies on top of an erodible bed material, the horseshoe vortex (HV) system plays an important role in the evolution of the scour hole around this structure.

Although there are many studies that tried to predict the scouring pattern and maximum scour depth for different hydraulic conditions and shapes of the structure (for a review see Melville, 1997), less effort has been made to understand the intricate flow physics behind the local scouring process and, in particular, the role played by the large-scale coherent structures present around the bridge abutments.

The turbulent horseshoe vortex system that forms around the bridge abutment is unsteady as its size, intensity and location changes with time. HV system is responsible for the increase in the turbulent kinetic energy and pressure r.m.s. fluctuations around its core locations along its trajectory. Furthermore HV system induces large bed shear stress and pressure r.m.s. fluctuations on the bed.

Koken and Constantinescu (2008a, 2008b) provided the first in-depth study of the structure and the unsteady dynamics of the flow field forming around vertical 
wall abutments both for flat bed and deformed bed conditions. They used a well resolved Large Eddy Simulation (LES) to simulate the flow field around the vertical wall abutment at a Reynolds number of 18,000 for both flat bed and deformed bed conditions. Flat bed conditions corresponded to the initiation of the scouring process and the deformed bed conditions represented the equilibrium scour conditions.

Although the initial and final stages of the scour are investigated in their study Koken and Constantinescu (2008a, 2008b) did not consider the intermediate stages. It is not clear how the coherent structures forming around the abutment changes throughout the scouring process. In the present study for a selected abutment length, the coherent structures, (especially the HV system) forming around the abutment are investigated at initial and two intermediate stages of the scour using DES.

\section{EXPERIMENTAL SETUP}

Experiments used in this study are performed in a $25 \mathrm{~m}$ long laboratory sediment flume which has width and height of $1.5 \mathrm{~m}$ and $0.5 \mathrm{~m}$, respectively. Sand particles with a median size of $\mathrm{d}_{50}=1.5 \mathrm{~mm}$ was used in the experiments. Sediment compartment of the channel, which is $6 \mathrm{~m}$ long, was filled with this material. These particles were also stuck on the channel surface to maintain uniform roughness. An abutment which has a $10 \mathrm{~cm}$ diameter semi-circular end was attached to one of the sidewalls within the sediment compartment section of the channel. The total length of the abutment including the semi-circular end was $15 \mathrm{~cm}$. Mean approach velocity, $\mathrm{U}$, and the flow depth, D, are selected as $0.335 \mathrm{~m} / \mathrm{s}$ and $0.135 \mathrm{~m}$, respectively, to ensure clear water scour conditions within the channel. The channel Reynolds number obtained using the mean approach velocity and flow depth was around 45,000 . Experiment was started and run for 2 hours. Then 3D scour patterns forming around the abutment were measured using an array of acoustic transducers along a grid refined up to 1-2 cm spacing. After this, experiment was run for another 2 hours which continued with the similar bathymetric measurements. Obtained bathymetric data together with the typical scour and deposition dimensions are given for both scour stages in Figure-1.

\section{NUMERICAL MODEL}

Detached Eddy Simulation is used to simulate the flow forming around the isolated bridge abutment which was described above. No wall functions are used in the simulations. The code uses fractional step method to integrate 3-D incompressible Navier-Stokes equations. Generalized curvilinear coordinates are used on non-staggered grid. Convective terms in the momentum equations are discretized using a blend of fifth-order accurate upwind biased scheme and secondorder central scheme. All other terms in the momentum and pressure-Poisson equations are approximated using second-order central differences. The discrete momentum (predictor step) and turbulence model equations are integrated in pseudotime using alternate direction implicit (ADI) approximate factorization scheme. In the present DES simulation, the Spalart-Allmaras (SA) one-equation model was used. Time integration in the DES code is done using a double time-stepping algorithm and local time stepping is used to accelerate the convergence at each 
physical time step. The time discretization is second order accurate. A general description of the code used in this study is given by Constantinescu and Squires (2004).

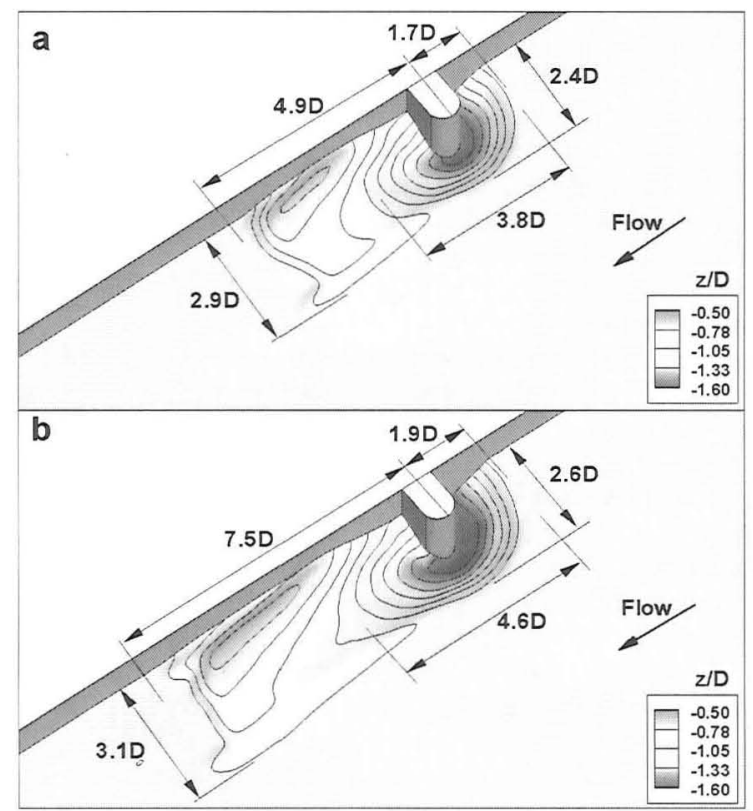

Figure-1: Scour and deposition pattern around the abutment obtained experimentally after: a) 2 hours (stage-1); b) 4 hours (stage-2).

All the parameters are normalized using the mean approach velocity $\mathrm{U}$, and flow depth $\mathrm{D}$ for the simulations. One flat bed (initiation of scour) and two deformed bed (intermediate stages of scour) simulations are conducted for the same abutment. The bathymetry data obtained from the experiments are used in the latter two simulations. Simulation performed at two hour scour conditions will be called as stage-1 and the one performed at four hour scour conditions will be called as stage-2 throughout the rest of the text. For all three simulations, total length of the domain was $38 \mathrm{D}$ where the abutment was placed at $8 \mathrm{D}$ distance from the inlet section. Consistent with the experimental model, flow domain had a width of 11.11D. Abutment has a width of $0.74 \mathrm{D}$ and a length of 1.11D including the semi-circular end. The domain is meshed using more than 4 million computational nodes. Nondimensional grid spacing along all the solid boundaries in the wall normal direction was $\Delta \mathrm{y}^{+}=\mathrm{yu}_{\tau} / \mathrm{v} \sim 1$ wall units (assuming that $\mathrm{u}_{\tau} / \mathrm{U}=0.04$, see Figure-2). At the inlet section a fully developed velocity profile which contained turbulent fluctuations are introduced in a time accurate fashion. This data is previously obtained from another 
Large Eddy Simulation performed at the same Reynolds number in a periodic straight channel and stored in a file. A convective boundary condition is used at the outlet which allowed the turbulent structures to leave the domain in a time accurate fashion without producing unphysical oscillations. Free surface is treated as a rigid lid. This is a reasonable assumption since the Froude number is $\sim 0.29$ within the experiments.

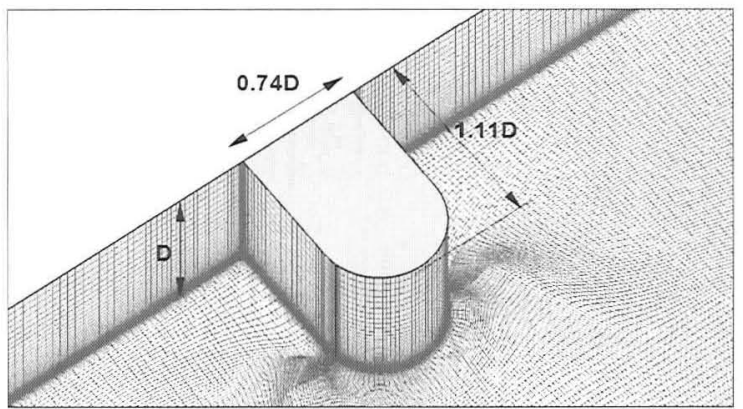

Figure-2: Abutment dimensions and typical computational mesh around it.

\section{RESULTS}

\section{Horseshoe Vortex System}

Coherent structures at the upstream of the abutment together with the horseshoe vortex system that wraps around the abutment are visualized using Qcriterion in Figure 3 for flat bed conditions and at two different scour stages (note that separated shear layers are covered in this figure). At the initiation of the scour (see Figure-3a) there are three horseshoe vortices which wrap around the base of the abutment together with a vertically oriented corner vortex, CV1, at the upstream recirculation region. Similar to the findings of Koken and Constantinescu (2008a), this corner vortex originates at the free surface and bends in the lateral direction as the bed is approached, where it merges with HV1. As a result it convects additional fluid and momentum from the regions close to the free surface into the core of HV1.

Main horseshoe vortex, HV1, originates close to the lateral wall and follows the upstream abutment face until the tip of the abutment where it bends in the flow direction. Past the abutment this vortex rapidly diffuses within the flow filed. On the other hand, secondary horseshoe vortex HV2 is larger in size and it is more coherent compared to HV1 and HV3. In the flow direction HV2 preserves its coherence up to a distance of $17 \mathrm{D}$ away from the abutment axis.

At scour stage-1, with the formation of the scour hole around the abutment, not only new coherent structures are observed around the abutment but also new interactions arise among these vortices (see Figure-3b). At this stage a secondary corner vortex CV2 is observed as well as the main corner vortex CV1 inside the upstream recirculation region. CV2 is located at the upstream of the main corner vortex, CV1, and is smaller in size compared to it. CV1 continues to feed the main 
necklace vortex HV1, whereas the secondary corner vortex CV2 feeds HV2 in a similar manner.

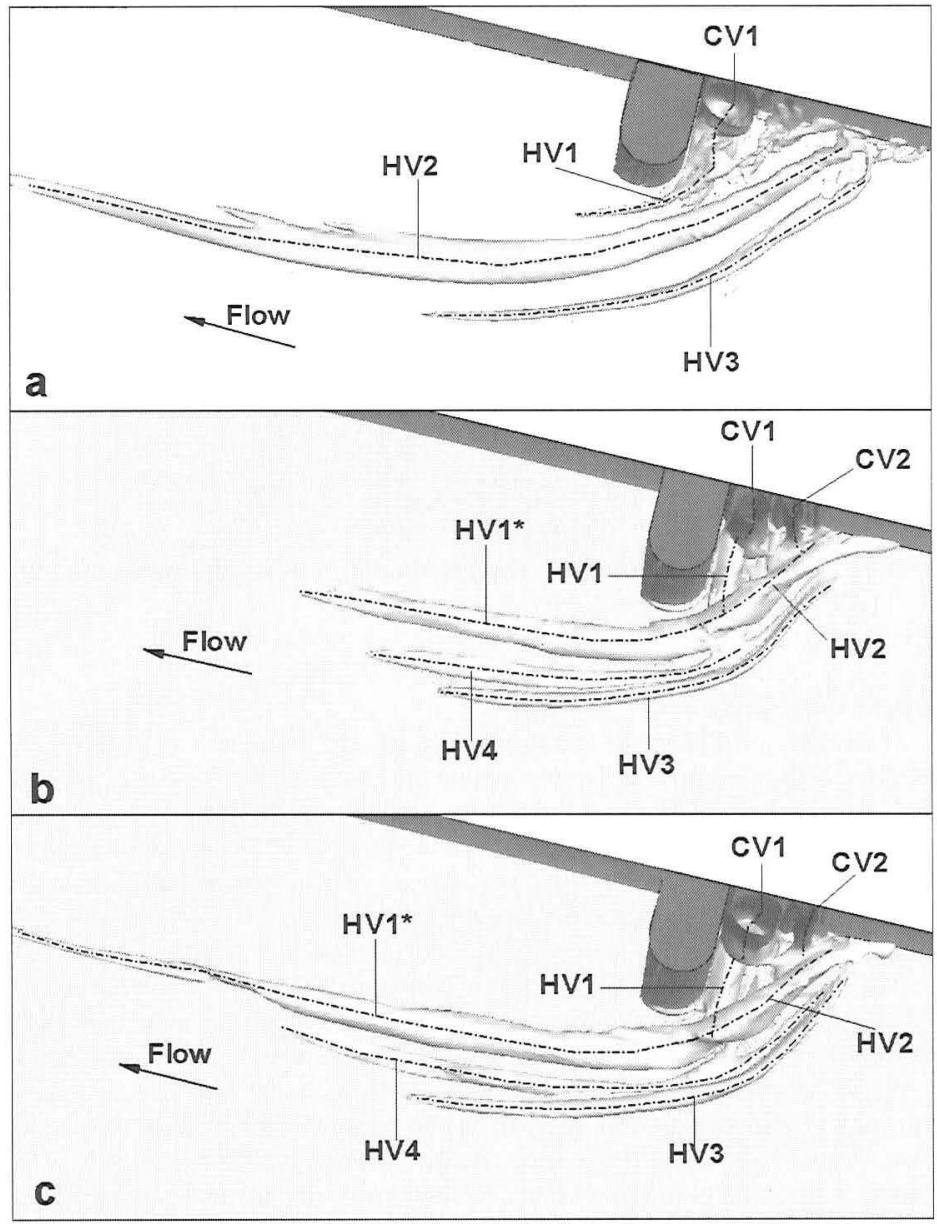

Figure-3: Vortical structures around the abutment for the mean flow (DES) visualized by $Q$ criterion for: a) Flat bed; b) Scour stage-1; c) Scour stage-2. (HV: horseshoe vortex, CV: corner vortex)

Secondary and tertiary horseshoe vortices HV2 and HV3 get closer to the abutment at this stage. At the upstream of the abutment HV1 and HV2 are confined within the scour hole whereas HV3 follows its edge. With the formation of the scour hole, a new counter rotating horseshoe vortex HV4 forms in between HV2 and HV3 
close to the bed. This vortex pushes HV2 away from the bed (see Figure-4b). As a result this vortex gets closer to the main horseshoe vortex HVl and starts interacting with it. At a location close to the tip of the abutment HV1 and HV2 merges into one vortex which will be called as $\mathrm{HV}^{*}{ }^{*}$ (see Figure-4c). Past the abutment HV1* follows the upslope and gets away from the bed. At a distance approximately 3.5D downstream of the abutment axis, core of $\mathrm{HV}^{*}$ * reaches mid-flow depth.

Compared with the first scour stage at stage-2 scour hole gets approximately $8 \%$ and $21 \%$ larger in the lateral and streamwise directions respectively (see Figure1). Moreover, the maximum scour depth measured from the free surface increases from $1.70 \mathrm{D}$ to $1.85 \mathrm{D}$. However the horseshoe vortex system is very similar to the one observed in stage-1. The only difference is the length scale over which $\mathrm{HV}^{*}$ * remains coherent. At stage- $1 \mathrm{HV}^{*}$ remains coherent up to a distance of $4 \mathrm{D}$ from the abutment axis in the downstream direction whereas in stage- $2 \mathrm{HV}^{*}$ is coherent up to 7D away from the abutment axis. This is related to the difference in the bathymetry at different scour stages, especially the steepness of the upslope at the downstream of the abutment (see Figure-1). Since at stage-1 the magnitude of this slope is larger, $\mathrm{HV}^{*}{ }^{*}$ gets away from the channel bed at a shorter length and diffuses within the flow quickly.

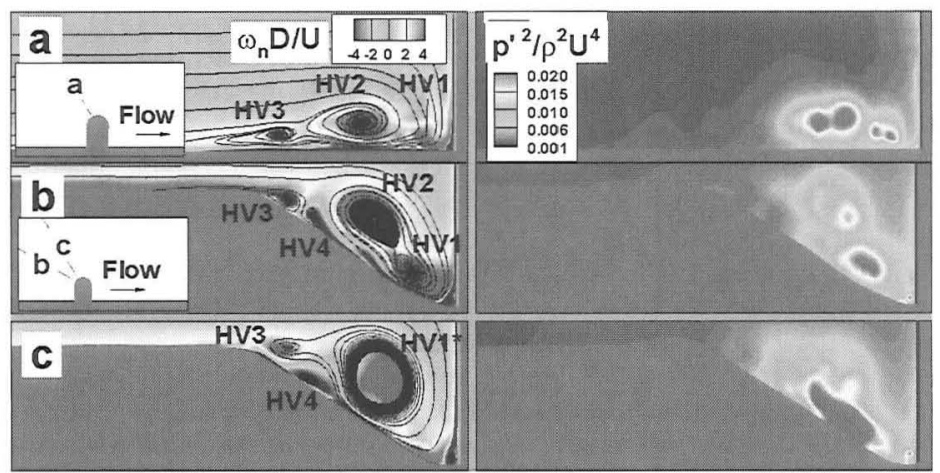

Figure-4: Mean out of plane vorticity contours together with the streamline patterns and resolved pressure r.m.s. fluctuations in representative vertical sections for: a) Initial scour stage; b) Scour stage-2; c) Scour stage-2. (see insets in $a$ and $b$ for plane locations)

Figures 4 show the streamline patterns superimposed on normalized out-ofplane vorticity contours, $\omega_{\mathrm{n}} \mathrm{D} / \mathrm{U}$, and the distribution of the normalized pressure r.m.s. fluctuations $\overline{p^{\prime 2}} /\left(\rho^{2} U^{4}\right)$ on vertical planes which cut through the HV system at different stages of scour. Here $\omega_{n}$ is the out of plane vorticity, whereas $\rho$ is the density. Throughout all the scour process pressure r.m.s. fluctuations are amplified within the core location of the primary and secondary necklace vortices. Moreover, at the initial stage of scour both for HV1 and HV2 r.m.s. pressure fluctuations show a double peaked distribution (see Figure-4a). This is a consequence of the bimodal 
nature of the flow within the main and secondary horseshoe vortices. The presence of large scale aperiodic oscillations were identified within the HV system forming around junction flows for the first time by Devenport and Simpson (1990). Koken and Constantinescu (2008a and 2008b) also identified this kind of oscillations for vertical wall abutment flows. However this is the first time these bimodal oscillations are observed both for the main and the secondary horseshoe vortices. The main reason for HV2 to have this bimodal behavior just like HV1 is that the core of HV2 is close enough to the abutment wall to get affected by it. Once the scour hole starts forming, as discussed earlier, HV2 is pushed towards HV1 because of the presence of a counter-rotating horseshoe vortex HV4 beneath HV2 close to the bed (see Figure-4b). At this section the bimodal nature is still present for HV1 (see the elongated amplification region within the core location of HV1). Even after the merging of HV1 and HV2 bimodal oscillations are observed within the core of $\mathrm{HV}^{*}$ * at sections close to the abutment tip (see Figure-4c).

\section{Bed Shear Stress}

The distribution of the normalized mean bed shear stress at initial stage and two later stages of the scour are given in Figure-5. At the initial stage, large bed shear stress values are observed in the acceleration region close to the tip of the abutment and beneath the horseshoe vortices (especially beneath HV1). Maximum normalized mean bed shear stress is measured as 0.015 at a location close to the tip of the abutment. For the experimental flow conditions the critical normalized bed shear stress is obtained from the Shields diagram as $\tau_{w c} /\left(\rho U^{2}\right)=0.007$. Possible scour regions where $\tau_{\mathrm{w}}$ is larger than $\tau_{\mathrm{wc}}$ are located at the upstream of the abutment and beneath the upstream part of the separated shear layers and the main horseshoe vortex HV1 (see Figure 5a).

At scour stage-1, with the formation of the scour hole at distances close to the bed, separated shear layers tend to curve behind the abutment. Because of this mechanism, some of the separated shear layer eddies tend to be entrained within the recirculation region. As a result, large bed shear stress values are observed at the downstream part of the abutment close to its tip. Also beneath the position where HV1 and HV2 merges, large bed shear stress values are observed. The maximum normalized mean bed shear stress value observed at stage- 1 and stage- 2 is around 0.01 and there is only a very small region at the downstream of the abutment where normalized mean bed shear stress is larger than $\tau_{\mathrm{wc}} /\left(\rho \mathrm{U}^{2}\right)=0.007$. However this critical value is obtained for flat bed conditions and has to be modified to take into account the bed slope effects. This is done by the formulation provided by Brooks and Shukry (1963), and the corrected normalized critical bed shear stress, $\tau_{\mathrm{wc}}{ }^{*} /\left(\rho \mathrm{U}^{2}\right)$, for sediment entrainment is calculated at every point on the bed. Obtaining this value, distribution of $\tau_{\mathrm{w}} / \tau_{\mathrm{wc}}{ }^{*}$ is plotted over the bathymetry both at stage- 1 and stage-2 (see Figure-6). Locations where this ratio is larger than 1 (which is enclosed by solid lines in Figure-6) represents the possible scour regions. Consistent with the experimental observations, the scour occurs mostly at the upstream of the abutment along the walls of the scour hole at two separate locations. There is also a small region at the base of the scour hole which is exposed to erosion. Compared with 
stage- 1 , one can see the considerable decrease in the possible erosion area at stage- 2 . This is logical as stage- 2 is closer to the equilibrium scour conditions at which there is practically no erosion $\left(\tau_{\mathrm{w}} / \tau_{\mathrm{wc}}{ }^{*}\right.$ is always less than 1$)$.

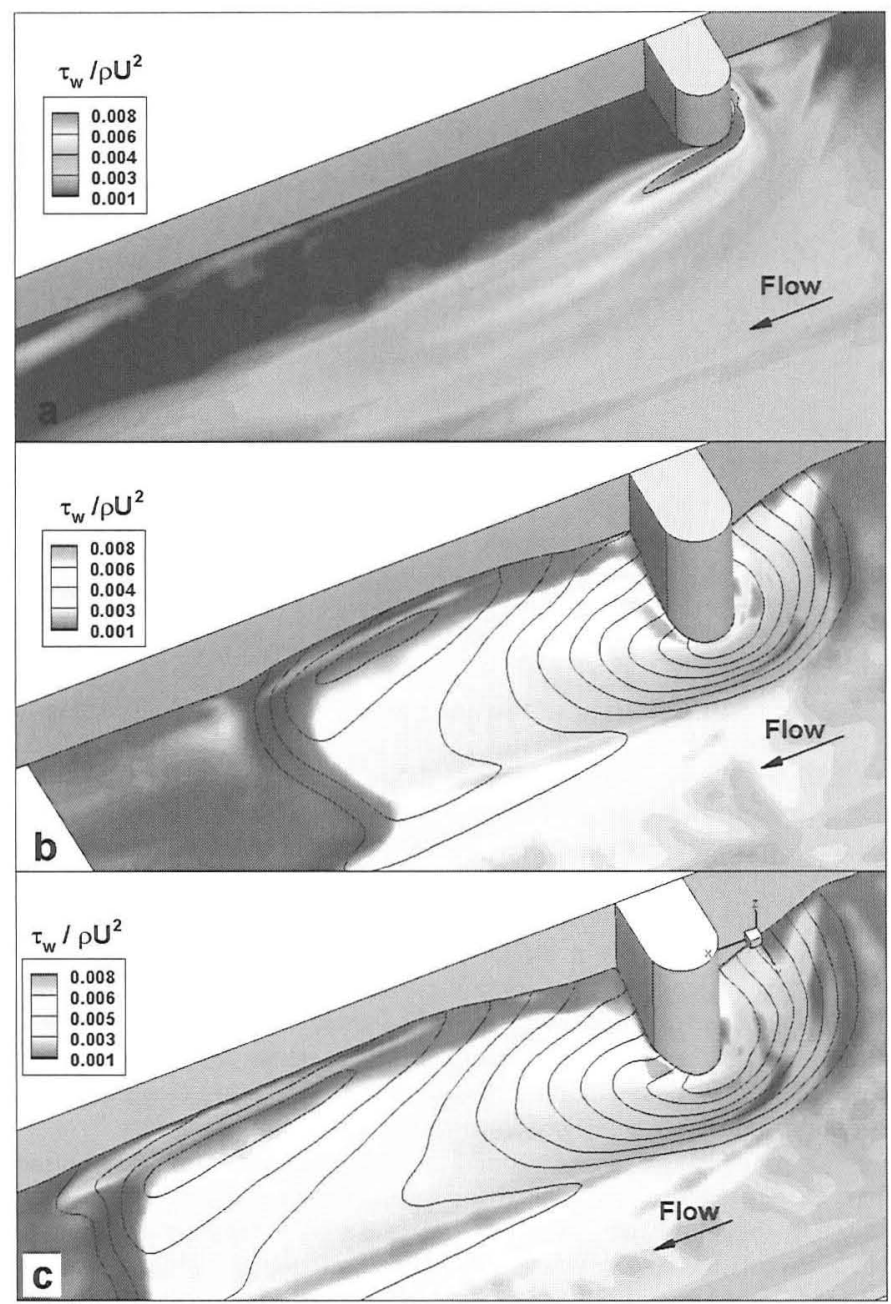

Figure-5: Distribution of the normalized mean bed shear stress for: a) Flat bed (solid line is $\tau_{w} / \rho U^{2}=0.007$ ); b) Scour stage-1; c) Scour stage-2. 


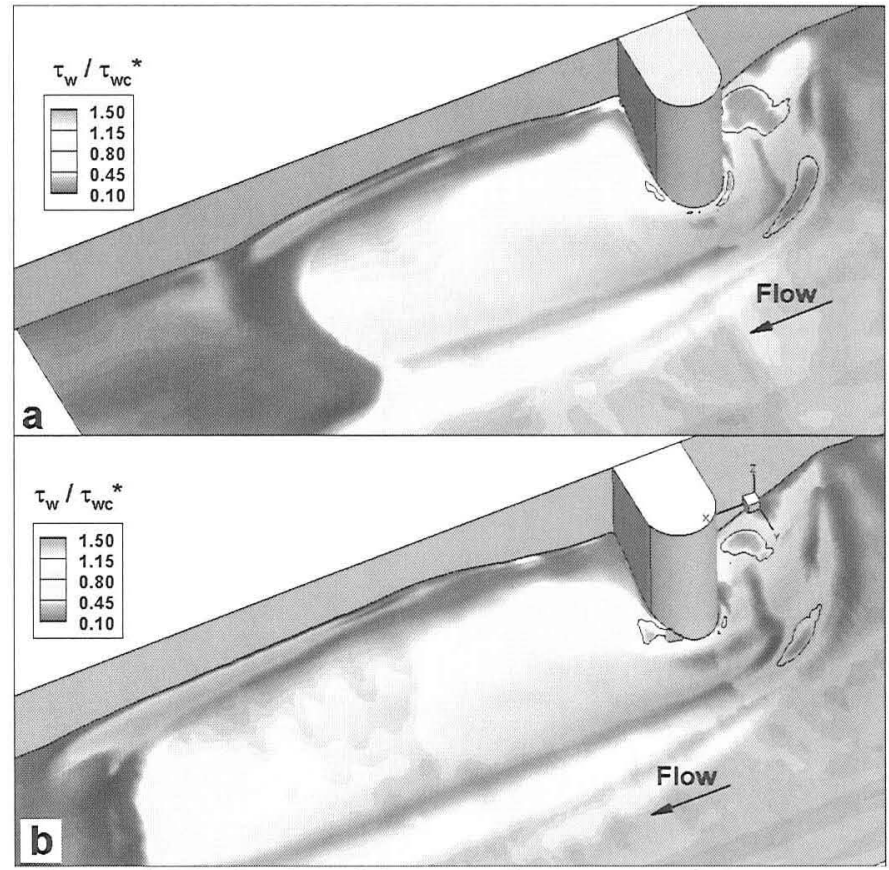

Figure-6: Distribution of the normalized mean bed shear stress

\section{CONCLUSIONS}

DES is used in the present study to investigate the changes in the coherent structures and especially the horseshoe vortex system at different stages of the scour process. At the initial stage of the scour, main horseshoe vortex is smaller in size and diffuses rapidly within the flow filed while the secondary necklace vortex is larger in size and remains coherent for a longer length in the streamwise direction. At this stage both of the vortices undergo aperiodic bimodal oscillations. Those oscillations cause the horseshoe vortices to induce large bed shear stress values beneath them. As the scour hole starts forming secondary necklace vortex HV2 gets closer to the primary necklace vortex HV1 and merges with it at a location close to the abutment tip. Bimodal oscillations are still present for this newly formed vortex.

At the initial stage of the scour bed shear stress values are obtained to be larger than the critical value required for sediment entrainment in the acceleration region close to the tip of the abutment and especially beneath the main necklace vortex HV1. This is the region where erosion starts forming. As the scour hole starts developing, at stage-1 mainly at two locations shear stress values exceed critical bed shear stress. These regions lay on the downslope of the scour hole at the downstream of the abutment and at the location where the scour hole is curved in the flow direction. This explains how the scour hole grows in the lateral and longitudinal 
directions. As the erosion continues at stage-2 at the same locations shear stress values are still larger than the critical value. However the area of these regions are considerably smaller. This is an expected result as the flow is approaching to the equilibrium scour conditions.

Since the changes in the structure of the horseshoe vortex system is not very different at stage-1 (2 hour scour) and stage-2 (4 hour scour); as a next step, a new experiment and a simulation will be held i.e. for 8 hour scour conditions.

\section{ACKNOWLEDGEMENTS}

This project is supported by TUBITAK (The Scientific and Technological Research Council of Turkey) under Project No: 108M590 and the computational resources used in this study were partly provided by TUBITAK ULAKBIM High Performance Computing Center, which are gratefully acknowledged here.

\section{REFERENCES}

Brooks, N. H., and Shukry, A. (1963). Discussion of "Boundary shear stress in curved trapezoidal channels." by A. T. Ippen and P. A. Drinker, Proc. ASCE, Journal Hydraulics Division, 89, HY3, 327-333.

Devenport, W. J., Simpson, R. L. 1990. Time-dependent and time-averaged turbulence structure near the nose of a wing-body junction. J. Fluid Mech., 210, 23-55.

Melville, B. W. (1997). "Pier and abutment scour: integrated approach." Journal of Hydraulic Engineering, Vol. 123(2), 125-136.

Constantinescu, G. and Squires, K. (2004). "Numerical investigations of flow over a sphere in the subcritical and supercritical regimes." Phys. of Fluids, 16(5), pp. 1449-1466, 2004.

Koken, M. and Constantinescu, G. (2008a). "An investigation of the flow and scour mechanisms around isolated spur dikes in a shallow open channel. Part I. Conditions corresponding to the initiation of the erosion and deposition process." Water Resources Research, 44, W08406, doi:10.1029/2007WR006489.

Koken, M. and Constantinescu, G. (2008b). "An investigation of the flow and scour mechanisms around isolated spur dikes in a shallow open channel. Part II. Conditions corresponding to the final stages of the erosion and deposition process." Water Resources Research, 44, W08407, doi:10.1029/2007WR006491. 